\title{
Indicadores Psicológicos e Comportamentais na Perícia do Abuso Sexual Infantil
}

\author{
Luiziana Souto Schaefer ${ }^{*}, 1,2$ \\ Orcid.org/0000-0002-7349-6532 \\ Alice Einloft Brunnet ${ }^{1,4}$ \\ Orcid.org/0000-0001-5290-1343 \\ Beatriz de Oliveira Meneguelo Lobo ${ }^{3}$ \\ Orcid.org/0000-0001-8774-2012 \\ Janaína Castro Núñez Carvalho ${ }^{1}$ \\ Orcid.org/0000-0001-5979-339X \\ Christian Haag Kristensen ${ }^{1}$ \\ Orcid.org/0000-0002-8273-2146
}

${ }^{1}$ Pontificia Universidade Católica do Rio Grande do Sul, Porto Alegre, RS, Brasil ${ }^{2}$ Instituto-Geral de Perícias do Rio Grande do Sul, Porto Alegre, RS, Brasil

${ }^{3}$ Centro Universitário Ritter dos Reis, Porto Alegre, RS, Brasil

${ }^{4}$ Université de Bourgogne Franche-Comté, Dijon, France

\section{Resumo}

Este estudo avaliou a capacidade discriminante de indicadores psicológicos e comportamentais frequentemente associados ao abuso sexual infantil. A amostra foi constituída por 79 crianças, de ambos os sexos, sendo $63,3 \%$ do sexo feminino $(n=50)$, com idades entre oito e 12 anos $(M=9,92 ; D P=1,45)$. Os participantes foram distribuídos em três grupos: Abuso sexual $(n=34)$, Maus-tratos sem histórico de abuso sexual $(n=14)$ e Sintomas clínicos sem histórico de maus-tratos $(n=31)$. Foram administrados instrumentos com os responsáveis e com as crianças. Para identificar os fatores que diferenciavam os grupos, foi realizada a Análise de regressão logística multinominal. A variável Preocupações sexuais foi significativa para diferenciar o grupo Abuso sexual dos outros dois grupos. O modelo apresentou capacidade preditiva geral de classificar corretamente $69,6 \%$ dos casos. Sugere-se que a investigação dessa variável seja incluída, entre outros indicadores, nos procedimentos periciais de crianças com suspeita de abuso sexual. Com base nos resultados obtidos, fica evidente que é necessário cautela no estabelecimento de associações causais entre manifestações comportamentais ou psicológicas e a hipótese de abuso sexual em crianças.

Palavras-chave: Abuso sexual na infância, sintomas comportamentais, sintomas psíquicos.

* Endereço para correspondência: Instituto-Geral de Perícias do Rio Grande do Sul, Departamento MédicoLegal, Avenida Ipiranga, 1807, Praia de Belas, Porto Alegre, RS, Brasil 90160093. Fone: (51) 32882654 E-mail: luiziana.schaefer@gmail.com, brunnetalice@gmail.com, beatrizlobo.m@gmail.com, janainanunez@ gmail.com e christian.kristensen@pucrs.br

Financiamento: Coordenação de Aperfeiçoamento de Pessoal de Nível Superior; Pontifícia Universidade Católica do Rio Grande do Sul; Instituto-Geral de Perícias do Rio Grande do Sul; Conselho Nacional de Desenvolvimento Científico e Tecnológico. 


\title{
Psychological and Behavioral Indicators in the Forensic Assessment of Child Sexual Abuse
}

\begin{abstract}
This study evaluated the discriminative ability of behavioral and psychological indicators often associated with child sexual abuse. The sample consisted of 79 children of both genders, $63.3 \%$ being female $(n=50)$, aged between 8 and 12 years $(M=9.92, S D=1.45)$. Participants were assigned to three groups: Sexual abuse $(n=34)$, Maltreatment with no history of sexual abuse $(n=14)$ and Clinical symptoms with no history of abuse or other traumatic events $(n=31)$. Assessment measures were administered with the legal guardians and with the children. A multinomial logistic regression analysis was conducted aiming to identify factors for differentiation of the groups. The TSCC Sexual concerns variable emerged as significant in differentiating the Sexual abuse group from both the Maltreatment and Clinical symptoms groups. The model showed overall predictive ability to accurately classify $69.6 \%$ of the cases. The investigation of this variable should be included, among other indicators, in forensic procedures for children with suspected sexual abuse. Based on the results, it becomes clear that caution is required in establishing causal relationships between behavioral or psychological manifestations and the hypothesis of child sexual abuse.
\end{abstract}

Keywords: Child sexual abuse, behavioral symptoms, psychic symptoms.

\section{Indicadores Psicológicos y Conductuales en la Pericia del Abuso Sexual Infantil}

\section{Resumen}

Este estudio evaluó la capacidad discriminante de indicadores psicológicos y conductuales frecuentemente asociados al abuso sexual infantil. La muestra fue constituida por 79 niños, de ambos sexos, siendo $63,3 \%$ do sexo femenino $(n=50)$, com edades entre ocho y 12 años $(M=9,92 ; D E=1,45)$. Los participantes fueron distribuidos en tres grupos: Abuso sexual $(n=34)$, Malos tratos sin antecedentes de abuso sexual $(n=14)$ y Sintomas clínicos sin antecedentes de maltrato $(n=31)$. Se han administrados instrumentos con los responsables y los niños. Para identificar los factores que diferenciaban a los grupos, se realizó el Análisis de regresión logística multinominal. La variable Preocupaciones sexuales apareció como significativa para diferenciar el grupo Abuso sexual de los otros dos grupos. El modelo presentó la capacidad preditiva general de clasificar correctamente $69,6 \%$ de los casos. Se sugerie la inclusión de esta variable, entre otros indicadores, en la evaluación del abuso sexual infantil. En base a los resultados obtenidos, es evidente que es necesario cautela en el establecimiento de asociaciones causales entre manifestaciones comportamentales o psicológicas y la hipótesis de abuso sexual infantil.

Palabras clave: Abuso sexual infantil, síntomas conductuales, síntomas psíquicos.

O abuso sexual infantil, como um problema social, de saúde e segurança pública, ainda representa um desafio em termos de avaliação, intervenção e prevenção. É definido como qualquer contato ou interação entre uma criança ou adolescente e alguém em estágio mais avançado de desenvolvimento psicossexual que usa a vítima para estimular-se sexualmente (World
Health Organization [WHO], 2002). O abuso sexual infantil apresenta dinâmica própria, caracterizada muitas vezes por segredos, ameaças, retratações, inexistência de testemunhas oculares, entre outros, sobretudo nos casos intrafamiliares (Peixoto, 2012). Tais características podem dificultar a revelação por parte da vítima, impactando a correta constatação do abuso 
sexual e, por conseguinte, a estimativa da sua real prevalência.

Em um estudo de metanálise foi encontrada uma prevalência total de $11,8 \%$ de abuso sexual na infância, sendo $18 \%$ entre o sexo feminino e 7,6\% entre o sexo masculino (Stoltenborgh, van IJzendoorn, Euser, \& Bakermans-Kranenburg, 2011). Quanto aos dados nacionais, foram recebidas, nos anos de 2015 e 2016, 37 mil casos de denúncias de violência sexual envolvendo vítimas na faixa etária de zero a 18 anos pelo Disque 100 (Portal Governo do Brasil, 2017). Convém mencionar que no Brasil ainda não há dados confiáveis sobre a prevalência do abuso sexual, portanto, provavelmente tais registros não representam a realidade dos casos de notificação no país.

Crianças sexualmente abusadas tendem a apresentar mais sintomatologia clínica do que aquelas que não passaram por esta experiência (Paolucci, Genuis, \& Violato, 2001). Por exemplo, o abuso sexual tem sido associado tanto a sintomas externalizantes (comportamentos deliquentes e agressividade) quanto a sintomas internalizantes (depressão, ansiedade, isolamento, dificuldades de atenção e queixas somáticas) (van den Heuvel \& Seedat, 2013). Ao mesmo tempo, não há um único sintoma que caracterize exclusivamente as vítimas e cerca de um terço das crianças que experienciaram abuso sexual não apresentam quaisquer sintomas (Kendall-Tackett, Williams, \& Finkelhor, 1993). No estudo realizado por Kendall-Tackett et al. (1993), dois clusters de sintomas foram identificados como os mais seguros para caracterizar as crianças vítimas de abuso sexual: comportamentos sexuais e sintomas relacionados ao Transtorno de Estresse Pós-traumático (TEPT). De maneira geral, estudos apontam que de um terço a metade de todas as crianças vítimas de abuso sexual apresentam sintomas pós-traumáticos clínicos significativos (Collin-Vézina, Daigneault, \& Hébert, 2013).

Apesar de não existir uma síndrome específica que caracteriza as vítimas (Paolucci et al., 2001), as consequências decorrentes do abuso sexual variam desde efeitos mínimos até problemas mais graves, com repercussões sociais, emocionais e/ou psiquiátricas (Habigzang, Bor- ges, Dell'Aglio, \& Koller, 2010; Kendall-Tackett et al., 1993; Paolucci et al., 2001; Wherry, Baldwin, Junco, \& Floyd, 2013). Especificamente, comportamentos sexuais inadequados (querer tocar os genitais de outras pessoas, demonstrar interesse e conhecimento sexual avançado para a idade, masturbar-se frequentemente e publicamente, entre outros) e preocupações sexuais (pensamentos de cunho sexual, sensações atípicas para a fase do desenvolvimento da criança ou que ocorrem em uma frequência maior que a esperada, conflitos sexuais, respostas negativas a estímulos sexuais e medo de ser sexualmente explorado) são alguns fatores mais diretamente referidos como sugestivos de abuso sexual (Briere, 1996; Friedrich et al., 2001; Friedrich et al., 1992; Friedrich, Jaworski, Huxsahl, \& Bengtson, 1997).

No contexto forense, um dos maiores desafios enfrentado pelos profissionais é a identificação do abuso sexual quando não há evidências físicas ou biológicas, o que representa a maior parte dos casos encaminhados para a perícia médica (Joki-Erkkila, Niemi, \& Ellonen, 2014). Neste contexto em que a ausência de vestígios físicos e biológicos não implica, necessariamente, que o abuso sexual não tenha ocorrido, recursos complementares de avaliação são indispensáveis (Peixoto, 2012; Ribeiro \& Peixoto, 2013).

A avaliação psicológica forense ou perícia psicológica tem adquirido papel preponderante como recurso investigativo em casos de suspeita de abuso sexual (Gava, Pelisoli, \& Dell'Aglio, 2013; Schaefer, Rossetto, \& Kristensen, 2012). Todavia, é necessário cautela na realização desta avaliação, pois, no campo psicológico, não há um indicador único que determine se uma criança foi sexualmente abusada (Pelisoli, Dell'Aglio, \& Herman, 2016). Portanto, análises abrangentes são as mais indicadas (Everson \& Faller, 2012; Schaefer et al., 2012).

A partir da revisão da literatura, identificou-se uma preocupação crescente quanto à utilização de estratégias para a avaliação do abuso sexual, especialmente no contexto forense (Everson \& Faller, 2012; Gava et al., 2013). Historicamente, indicadores comportamentais e psicológicos utilizados na avaliação clínica pas- 
saram a ser igualmente aplicados na área forense. Tais critérios, muitas vezes empregados indiscriminadamente, acabavam por desconsiderar outros fatores no histórico e no contexto de vida do examinando que poderiam ter desencadeado e/ou contribuído no desenvolvimento do quadro sintomatológico (Gava et al., 2013). Diante disso, tais estratégias de avaliação passaram a ser rigorosamente questionadas, e pesquisadores da área investiram na elaboração de técnicas e procedimentos de avaliação cujos valores preditivos fossem menos controversos (Peixoto, 2012).

Esforços têm sido observados no desenvolvimento de protocolos investigativos de coleta $\mathrm{e}$ avaliação do relato da criança, já que este, muitas vezes, é um dos únicos elementos de prova (Peixoto, 2012; Peixoto et al., 2017; Williams, Hackbarth, Aznar-Blefari, Padilha, \& Peixoto, 2014). Ainda que haja consenso quanto à utilização da entrevista forense como elemento central, grande parte dos pesquisadores também sustenta que o uso de outros indicadores e de critérios objetivos de avaliação não podem ser desprezados neste contexto (Everson \& Faller, 2012). Além disso, conforme já mencionado, há evidências das consequências psíquicas, comportamentais, psicopatológicas e, até mesmo, neurobiológicas desencadeadas em vítimas de abuso sexual, com repercussões, inclusive, na idade adulta (Irigaray et al., 2013).

O presente estudo foi proposto considerando a premissa de que a avaliação do abuso sexual no contexto forense deve ser realizada de maneira integrada e compreensiva, incluindo não apenas a entrevista e a avaliação do relato da criança, mas também indicadores objetivos e válidos para este contexto e para esta população. Assim, esta pesquisa teve como principal objetivo avaliar indicadores psicológicos e comportamentais na perícia de crianças com suspeita de abuso sexual. Especificamente, buscou-se avaliar a capacidade discriminante das seguintes variáveis: comportamentos sexuais, cognições pós-traumáticas, sintomas externalizantes e internalizantes, sintomas de estresse pós-traumático, de depressão, de ansiedade, de dissociação, raiva e preocupações sexuais.

\section{Método}

\section{Participantes}

Participaram da pesquisa 98 crianças recrutadas a partir de serviços de saúde mental da cidade de Porto Alegre e no órgão de perícia oficial do estado do Rio Grande do Sul, Brasil. Os critérios de exclusão utilizados foram: (a) presença de sintomas psicóticos, avaliados através do Child Behavior Checklist (CBCL), (b) indicativo de déficit intelectual, obtido através do percentil $<5$ no Teste Matrizes Progressivas de Raven e (c) ter invalidado o instrumento Trauma Symptom Checklist for Children (TSCC; escores $t>=70$ na escala de hiporresponsividade e escores $t>=90$ na escala de hiperresponsividade). Foram excluídos da amostra 19 participantes: um deles apresentou sintomas psicóticos, quatro apresentaram indicativos de déficit intelectual e 14 invalidaram o TSCC (duas crianças teriam superestimado os sintomas e 12 crianças teriam subestimado os sintomas).

A amostra final foi constituída por 79 crianças, de ambos os sexos, sendo $63,3 \%$ do sexo feminino $(n=50)$, com idades entre oito e 12 anos $(M=9,92 ; D P=1,45)$. A maioria das crianças $(88,6 \% ; n=70)$ estudava em escolas públicas, $94,9 \%(n=75)$ possuíam ao menos um irmão $(M=2,28 ; D P=1,5), 23,1 \%(n=18)$ já repetiram alguma série, $57,7 \%(n=45)$ possuíam pais separados e $67,1 \%(n=53)$ pertenciam à classe econômica $\mathrm{C}$ ou $\mathrm{D}$, segundo critério de classificação econômica do Brasil (Associação Brasileira de Empresas de Pesquisa, 2015).

Os participantes foram distribuídos em três grupos conforme os critérios descritos a seguir. As crianças com abuso sexual confirmado através de perícia física ou psicológica, ambas realizadas pelo órgão da perícia oficial do estado do Rio Grande do Sul, foram alocadas no grupo Abuso sexual $(n=34)$. Destas, $41,2 \%(n=14)$ também vivenciaram ao menos outra forma de maus-tratos. As crianças que não tinham suspeita oficial de abuso sexual (denúncia) ou extra oficial (conforme investigado no momento da coleta de dados pelos pesquisadores deste estudo na entrevista semiestruturada com os responsáveis e 
com a própria criança), mas preencheram os critérios de vivência de maus-tratos e/ou violência doméstica conforme definição da WHO (2002) foram incluídas no grupo Maus-tratos sem histórico de abuso sexual $(n=14)$. Destas, $42,8 \%$ $(n=6)$ experienciaram mais de uma forma de maus-tratos e, entre aquelas que passaram por um único tipo, 21,4\% $(n=3)$ sofreram negligência, $14,3 \%(n=2)$ experienciaram abuso físico, $14,3 \%(n=2)$ vivenciaram violência doméstica e $7,1 \%(n=1)$ sofreram abuso emocional. Os participantes com sintomas clínicos avaliados pelo CBCL e sem qualquer histórico de maus-tratos ou de outros eventos traumáticos (conforme investigado na entrevista semiestruturada com os responsáveis e com a própria criança) foram alocados no grupo Sintomas clínicos sem histórico de eventos traumáticos $(n=31)$. O histórico de abuso sexual e de outros maus-tratos foi obtido pelos pesquisadores deste estudo através da entrevista semiestruturada com os responsáveis e do relato da criança.

\section{Instrumentos}

Instrumentos Respondidospelos Responsáveis:

Entrevista Semiestruturada com os Responsáveis: entrevista elaborada exclusivamente para esta pesquisa, realizada individualmente com os responsáveis nos moldes de uma entrevista semiestruturada, englobando questões sobre sexo, idade, escolaridade, nível socioeconômico e condições de saúde dos participantes. Também foi investigada a exposição da criança a situações de maus-tratos, conforme definição da WHO (2002), e outras experiências traumáticas, conforme definição do DSM-5 (American Psychiatric Association, 2014), em que o indivíduo é exposto a episódio concreto ou ameaça de morte, lesão grave ou violência sexual, seja de forma direta, indireta ou como testemunha.

Child Behavior Checklist (CBCL; Achenbach, 1991, versão brasileira por Bordin, Mari, \& Caeiro, 1995): inventário respondido pelos pais em relação aos comportamentos dos filhos, objetivando identificar aspectos comportamentais e emocionais de crianças entre 6 e 18 anos, além de possíveis transtornos psicopatológicos. Em todas as escalas do CBCL, a sintomatologia da criança é classificada como clínica, limítrofe ou não clínica, de acordo com a amostra normativa de pares de Achenbach (1991, 2001).

Child Sexual Behavior Inventory (CSBI; Friedrich et al., 1992, versão brasileira por Miele \& Arteche, 2017): questionário de 35 itens a serem respondidos pelos pais. Avalia a frequência de comportamentos sexuais específicos, abrangendo nove domínios: Limites interpessoais, Ansiedade sexual, Exibicionismo, Intromissão sexual, Representação de papéis sexuais, Conhecimento sexual, Auto-estimulação, Comportamento voyeurístico e Interesse sexual. O instrumento foi originalmente projetado e validado para a avaliação de crianças de dois a 12 anos de idade. É considerado o instrumento mais utilizado para a avaliação de comportamento sexual em crianças (Everson \& Faller, 2012). Estudos que empregaram o instrumento encontraram níveis clínicos significativos de comportamento sexualizado em aproximadamente 30 a $40 \%$ da amostra de crianças vítimas de abuso sexual, além de observarem diferenças significativas entre crianças sexualmente abusadas e aquelas que não vivenciaram esta experiência (Friedrich, 2007).

\section{Instrumentos para as Crianças:}

Entrevista semiestruturada com a criança: esta entrevista foi elaborada pelos pesquisadores e teve como objetivo estabelecer o vínculo com a criança e investigar a vivência de eventos traumáticos, incluindo situações de maus-tratos, e explorando o evento mais traumático. A entrevista foi realizada conforme orientações propostas pelo protocolo de Michigan (Faller, 2003), composta basicamente por: etapa inicial (rapport, estabelecimento das regras básicas da entrevista, avaliação do nível de desenvolvimento do avaliado, se a criança consegue distinguir realidade e fantasia, bem como verdade e mentira, e investigação geral de aspectos da sua vida); etapa focalizada no evento (com o uso de perguntas abertas, objetiva investigar a vivência de eventos traumáticos, estimulando a emissão do relato livre); e etapa do encerramento (informa-se a criança sobre as próximas etapas, colocan- 
do-se disponível, ajudando a criança a restabelecer o equilíbrio, incluindo a manifestação de sentimentos, pensamentos e atitudes em relação à revelação e a situação vivenciada).

Teste Matrizes Progressivas de Raven (Raven, Court, \& Raven, 1995) - Escala Geral (versão em português por Angelini, Alves, Custódio, Duarte, \& Duarte, 1999) e Escala Especial (versão em português por Angelini, Alves, Custódio, \& Duarte, 1987): constitui-se por um teste não-verbal para avaliação do nível de inteligência de indivíduos com a versão Geral (a partir dos 11 anos) e Especial (5-11 anos).

Trauma Symptom Checklist for Children (TSCC; Briere, 1996, versão brasileira por Lobo, Brunnet, Ecker, Schaefer, Arteche, \& Kristensen, 2015): mensura sintomas pós-traumáticos e sintomas psicológicos associados em crianças e adolescentes entre oito e 16 anos que experienciaram eventos traumáticos. É uma escala de auto-relato com 54 itens na qual se busca rastrear sentimentos, pensamentos e comportamentos, em que o sujeito preenche entre 0 (nunca) e 4 (o tempo todo) em uma lista abrangente de sintomas pós-traumáticos. Possui duas escalas de validade (hiporresponsividade e hiperresponsividade), seis escalas clínicas (ansiedade, depressão, raiva, estresse pós-traumático, dissociação e preocupações sexuais) e oito itens críticos (Briere, 1996) que avaliam aspectos que necessitam de atenção clínica imediata. Para todas as escalas clínicas, com exceção da escala de Preocupações sexuais, os escores $T$ iguais ou acima de 65 são considerados clinicamente significativos, e entre 60 e 65 sugerem sintomatologia subclínica significativa. Para a escala de Preocupações sexuais, escores $T$ iguais ou acima de 70 são considerados clinicamente significativos.

Child Post-Traumatic Cognitions Inventory (CPTCI; Meiser-Stedman et al., 2009, versão brasileira por Lobo, Brunnet, Ecker, Schaefer, Arteche, Gauer, et al., 2015): é uma escala de auto-relato composta por 25 itens, tendo como objetivo mensurar cognições pós-traumáticas negativas em crianças e adolescentes, com idades entre seis e 18 anos, em uma escala Likert entre 1 (não concordo) e 4 (concordo plenamente). Ela possui dois componentes: mudança per- manente e perturbadora após o trauma e pessoa frágil em um mundo assustador.

\section{Procedimentos Éticos e de Coleta dos Dados}

O presente estudo foi aprovado pelo Comitê de Ética em Pesquisa da Pontifícia Universidade Católica do Rio Grande do Sul (PUCRS), conforme consta no parecer $n^{\circ}$ 247.127. Foi obtido o Termo de Consentimento Livre e Esclarecido de todos os responsáveis legais pelas crianças e o Termo de Assentimento de todas as crianças que participaram do estudo, conforme estabelecido na Resolução no 466/2012 do Conselho Nacional de Saúde (2013). Os dados foram coletados em duas sessões de aproximadamente 1 hora com os responsáveis e duas sessões de aproximadamente 1 hora com as crianças. Os casos em que se constatou a necessidade de intervenção médica e/ou psicológica foram encaminhados para serviços de referência e os casos em que se avaliou risco atual de exposição a situações de maus-tratos foram comunicados às autoridades responsáveis.

\section{Procedimentos de Análise dos Dados}

As variáveis sociodemográficas e clínicas foram descritas através de medidas de tendência central e variabilidade. Foram observadas as frequências das variáveis, conforme os pontos de corte estabelecidos nos instrumentos. Foram conduzidas análises de diferença intragrupos na variável sexo, através do teste qui-quadrado, e também análises de diferenças entre os sexos na amostra geral, através do teste Mann-Whitney $U$, nas seguintes variáveis: cognições pós-traumáticas, comportamentos sexuais, sintomas internalizantes, sintomas externalizantes, estresse pós-traumático, ansiedade, depressão, raiva, dissociação e preocupações sexuais. Também foram realizadas análises de diferenças entre grupos, através do teste Kruskal Wallis, nos oito itens críticos do TSCC e nos nove domínios do CSBI.

Com o objetivo de identificar os fatores que diferenciavam os grupos, foi realizada a Análise de regressão logística multinominal. Foram 
realizados testes de multicolinearidade e todas as variáveis incluídas no modelo apresentaram Fatores de Inflação da Variância $(\mathrm{VIF})<5$, indicando a ausência de casos graves de multicolinearidade. A variável dependente foi o grupo: Abuso Sexual, Maus-tratos sem histórico de abuso sexual e Sintomas Clínicos sem histórico de eventos traumáticos. O grupo Abuso sexual foi utilizado como referência. As variáveis independentes incluídas foram: comportamentos sexuais, cognições pós-traumáticas, sintomas de estresse pós-traumático, de depressão, de ansiedade e de dissociação, raiva e preocupações sexuais. Devido às características da amostra, foram utilizados testes não-paramétricos. $\mathrm{O}$ nível de significância utilizado foi de 5\%. Os dados dos instrumentos foram computados e analisados no Statistical Package for Social Sciences (SPSS) for Windows, versão 17.0.

\section{Resultados}

A partir da análise da amostra geral, através do teste qui-quadrado, foram observadas diferenças estatisticamente significativas em relação ao percentual de meninos e meninas na amostra, com maior prevalência do sexo feminino $\left[\chi^{2}(1)\right.$ $=5,58 ; p=0,018]$. A partir dos resultados do teste Mann-Whitney $U$, foram encontradas diferenças estatisticamente significativas na amostra geral somente na variável sintomas externalizantes $[Z(79)=-2,98 ; p=0,003]$, com escores mais altos entre os meninos. $\mathrm{Na}$ análise intragrupos, através do teste qui-quadrado, a variável sexo apresentou diferença estatisticamente significativa somente no grupo abuso sexual, com maior prevalência de meninas $\left[\chi^{2}(1)=9,53 ; p=0,002\right]$.

Especificamente quanto ao grupo Abuso sexual, entre os casos válidos, $43,7 \%(n=14)$ das crianças vivenciaram episódio único e $56,3 \%$ ( $n$ $=18$ ) vivenciaram episódios recorrentes de abuso sexual. Além disso, em $38,2 \%(n=13)$ dos casos, os episódios foram perpetrados por algum membro da família nuclear (mãe/madrasta, pai/ padrasto, irmãos), 44,1\% $(n=15)$ por algum membro da família extensa (avós, tios, primos, amigos e vizinhos próximos), $14,7 \%(n=5)$ por conhecidos, mas não próximos, e em apenas um caso $(2,9 \%)$ o agressor era desconhecido da vítima. Quanto ao sexo dos perpetradores, em $94,1 \%(n=32)$ das ocorrências o agressor era do sexo masculino e em 5,9\% $(n=3)$ dos casos, os episódios foram perpetrados simultaneamente por homens e mulheres. Em 94,1\% dos casos $(n$ $=32$ ), houve algum tipo de contato físico (toques, carícias ou intercurso sexual) e em 5,9\% ( $n$ =2) não houve contato físico (exibição de genitais ou exposição a material pornográfico). Em $8,8 \%$ dos casos $(n=3)$ o resultado da perícia física foi positivo. Em $71,4 \%(n=15)$ das ocorrências houve ameaça e em $28,6 \%(n=6)$ dos casos foram caracterizados por persuasão não violenta. Por último, em 75,8\% $(n=25)$ dos casos, o último episódio de abuso sexual teria ocorrido há menos de um ano da data da coleta dos dados.

Foram realizadas análises de diferenças entre grupos, através do teste Kruskal Wallis, nos oito itens críticos do TSCC e nos nove domínios do CSBI. Os resultados estão apresentados na Tabela 1.

Com o objetivo de identificar os fatores que diferenciavam os grupos, foi realizada a Análise de regressão logística multinominal. Foram investigadas as seguintes variáveis: comportamentos sexuais, ansiedade, depressão, raiva, estresse pós-traumático, dissociação, preocupações sexuais, cognições pós-traumáticas, sintomas externalizantes e sintomas internalizantes. Os resultados estão apresentados na Tabela 2. O grupo Abuso sexual foi utilizado como referência.

Como pode ser visto, a variável Preocupações sexuais (TSCC) foi a única que apareceu como significativa para diferenciar o grupo Abuso sexual tanto do grupo Maus-tratos sem histórico de abuso sexual, como do grupo Sintomas clínicos sem histórico de eventos traumáticos. A partir dos resultados, é possível verificar que os participantes do grupo Maus-tratos têm $8 \%$ menos chance de apresentar preocupações sexuais do que o grupo Abuso sexual, ao passo que o grupo Sintomas clínicos tem $20 \%$ menos chance de apresentar preocupações sexuais do que o grupo Abuso sexual. Ao se utilizar a variável Preocupações sexuais para classificar os grupos, a porcentagem de casos identificados corretamente é de 73,5\% (25 em 34 casos) no grupo 
Tabela 1

Diferença entre Grupos nos Itens Críticos do TSCC e nos Domínios do CSBI

\begin{tabular}{|c|c|c|c|c|}
\hline & \multicolumn{3}{|c|}{ Média $(D P)$} & \multirow[b]{2}{*}{$X^{2}$} \\
\hline & $\begin{array}{l}\text { Maus-tratos } \\
\quad(n=14)\end{array}$ & $\begin{array}{l}\text { Abuso Sexual } \\
\qquad(n=34)\end{array}$ & $\begin{array}{l}\text { Sintomas Clínicos } \\
\qquad(n=31)\end{array}$ & \\
\hline \multicolumn{5}{|l|}{ Itens críticos TSCC } \\
\hline Medo de homens & $0,79(0,80)$ & $0,73(0,79)$ & $0,32(0,70)$ & $7,96^{*}$ \\
\hline Sentir medo de mulheres & $0,57(1,02)$ & $0,21(0,41)$ & $0,13(0,34)$ & 2,28 \\
\hline Querer machucar outras pessoas & $0,50(0,94)$ & $0,41(0,56)$ & $0,52(0,89)$ & 0,10 \\
\hline Querer machucar a mim mesmo & $0,50(0,52)$ & $0,23(0,43)$ & $0,32(0,54)$ & 3,16 \\
\hline Entrar em brigas & $1,29(1,27)$ & $0,65(0,73)$ & $0,58(0,76)$ & 3,49 \\
\hline Sentir medo de que alguém vai me matar & $0,86(1,10)$ & $0,68(1,04)$ & $0,71(0,97)$ & 0,49 \\
\hline Querer me matar & $0,36(0,84)$ & $0,15(0,36)$ & $0,16(0,37)$ & 0,44 \\
\hline $\begin{array}{l}\text { Não confiar nas pessoas porque elas podem } \\
\text { querer manter relações sexuais }\end{array}$ & $0,57(1,09)$ & $1,03(1,14)$ & $0,10(0,30)$ & $16,47 * *$ \\
\hline \multicolumn{5}{|l|}{ Domínios CSBI } \\
\hline Intromissão sexual & $0,29(0,61)$ & $0,38(0,74)$ & $0,03(0,18)$ & $6,72 *$ \\
\hline Conhecimento sexual & $0,57(1,16)$ & $0,94(1,57)$ & $0,03(0,18)$ & $9,36^{*}$ \\
\hline Comportamento voyeurístico & $1,5(1,99)$ & $0,71(1,36)$ & $0,16(0,45)$ & $7,23 *$ \\
\hline Limites interpessoais & $1,57(2,50)$ & $1,38(1,46)$ & $1,16(1,71)$ & 1,10 \\
\hline Ansiedade Sexual & $0,86(1,23)$ & $0,35(0,85)$ & $0,19(0,60)$ & 4,02 \\
\hline Exibicionismo & $0,14(0,53)$ & $0,06(0,24)$ & $0,13(0,56)$ & 0,04 \\
\hline Representação de papéis sexuais & $0,14(0,53)$ & $0,06(0,24)$ & $0,23(0,67)$ & 1,12 \\
\hline Auto-estimulação & $0,14(0,53)$ & $0,24(0,85)$ & $0,77(1,71)$ & 3,37 \\
\hline Interesse sexual & $0,64(0,74)$ & $0,68(1,27)$ & $0,45(0,99)$ & 2,48 \\
\hline
\end{tabular}

${ }^{*} p<0,05 ; * * p<0,001$.

Abuso sexual, 28,6\% (4 em 14 casos) no grupo Maus-tratos, e 83,9\% (26 em 31 casos), no grupo Sintomas clínicos. O modelo apresentou capacidade preditiva geral de classificar corretamente $69,6 \%$ dos casos.

\section{Discussão}

O objetivo geral do estudo foi avaliar indicadores psicológicos e comportamentais na perícia de crianças com suspeita de abuso sexual. A partir dos resultados encontrados, pode-se observar que o fator que diferenciou de forma significativa o grupo Abuso sexual dos demais grupos foi a variável Preocupações sexuais. As demais variáveis de depressão, ansiedade, estresse pós-traumático, dissociação, cognições pós-traumáticas, raiva e comportamentos sexuais, bem como as variáveis de sintomatologia global internalizante e externalizante não se mostraram discriminantes na diferenciação dos grupos. Para a compreensão das análises, é importante retomar que foram avaliados, de uma forma geral, a partir do CBCL, sintomas internalizantes (somatório das escalas de ansiedade/depressão, isolamento/depressão, queixas somáticas, problemas sociais, problemas de pensamento, problemas de atenção) e externalizantes (somatório 
Tabela 2

Regressão Logística Multinominal do Grupo Maus-Tratos e do Grupo Sintomas Clínicos em comparação ao Grupo Abuso Sexual

\begin{tabular}{lcccc}
\hline & \multicolumn{2}{c}{ Maus-Tratos } & \multicolumn{2}{c}{ Sintomas clínicos } \\
\cline { 2 - 5 } & Odds ratio & IC 95\% & Odds ratio & IC 95\% \\
\hline Comportamentos sexuais & 0,98 & $0,84-1,15$ & 0,91 & $0,785-1,06$ \\
Ansiedade & 1,04 & $0,94-1,15$ & 1,06 & $0,97-1,15$ \\
Depressão & 0,95 & $0,83-1,09$ & 1,06 & $0,93-1,22$ \\
Raiva & 1,14 & $1,00-1,29$ & 1,04 & $0,93-1,18$ \\
Estresse pós-traumático & 1,00 & $0,91-1,12$ & 0,94 & $0,85-1,04$ \\
Dissociação & 1,04 & $0,92-1,16$ & 1,07 & $0,96-1,20$ \\
Preocupações sexuais & 0,92 & $0,84-1,00^{*}$ & 0,80 & $0,71-0,90 * *$ \\
Cognições Pós-traumáticas & 0,96 & $0,88-1,04$ & 0,94 & $0,88-1,01$ \\
Sintomas internalizantes & 1,00 & $0,92-1,08$ & 1,01 & $0,93-1,09$ \\
Sintomas externalizantes & 1,06 & $0,98-1,16$ & 1,03 & $0,96-1,09$ \\
\hline
\end{tabular}

Nota. Grupo de Referência $=$ Abuso Sexual, $\chi^{2}(20)=46,63, p=0,001$.

${ }^{*} p<0,05 ; * * p<0,001$.

das escalas de comportamento de quebrar regras/ delinquente e comportamento agressivo) e a partir dos demais instrumentos foram analisadas escalas específicas de sintomas de depressão, ansiedade, estresse pós-traumático, dissociação, preocupações sexuais e cognições pós-traumáticas, que seriam conceitualizados como sintomas internalizantes, e escalas de raiva e de comportamentos sexuais, como externalizantes.

Em relação às análises descritivas, foi possível verificar que grande parte das crianças, tanto do grupo Abuso sexual (50\%) quanto do grupo Maus-tratos sem histórico de abuso sexual $(64,2 \%)$, vivenciaram outros eventos traumáticos ao longo da vida, sendo que os mais comuns referiram-se a outras situações de maus-tratos. A polivitimização, ou seja, a vivência de múltiplos eventos traumáticos parece ser uma realidade que atinge muitas crianças, dificultando a mensuração dos efeitos desta exposição, sobrepondo possíveis sintomas e prejuízos associados (Guerra, Ocaranza \& Weinberger, 2016; Hamby, Finkelhor \& Turner, 2014).

Quanto às análises das diferenças entre os sexos na amostra geral, a única variável que apresentou diferença estatisticamente significa- tiva foi sintomas externalizantes (quando avaliados globalmente pelo CBCL), com escores mais altos entre os meninos. Estes achados são corroborados pela literatura que aponta que, de maneira geral, os meninos tendem a apresentar mais sintomas externalizantes do que as meninas (Chaplin \& Aldao, 2013). Ao mesmo tempo, no presente estudo, não foram encontradas diferenças significativas quanto aos sintomas internalizantes. Pesquisas anteriores discutiram que embora as meninas sejam mais propensas do que os meninos a sofrerem abuso sexual, a vitimização não explicou o risco aumentado para sintomas internalizantes entre as meninas, sugerindo que sexo e vitimização devem ser considerados como fatores de risco independentes para a severidade dos sintomas internalizantes (Ruiz, 2016). Também não foram encontradas diferenças significativas nos sintomas pós-traumáticos entre meninos e meninas, corroborando os achados de estudos anteriores (Peixoto, 2012).

Ainda em relação às características gerais da amostra estudada, a maioria dos participantes apresentou sintomas externalizantes e internalizantes na faixa clínica ou limítrofe, quando tais construtos foram avaliados de maneira glo- 
bal no CBCL. Por outro lado, ao se considerar os pontos de corte estabelecidos para as escalas do TSCC, a maioria das crianças dos três grupos não apresentou sintomatologia significativa em todas as escalas desse instrumento. A partir destes achados, pode-se discutir o fato de que um dos instrumentos (CBCL) foi respondido pelos responsáveis e o outro (TSCC) pela própria criança. Em virtude disso, em especial no contexto forense, é importante considerar dados provenientes de diferentes fontes de informação, com medidas de auto e hetero-relato, uma vez que tanto os responsáveis quanto a própria criança podem não responder com exatidão ao que está sendo questionado, ora sub ou superestimando sintomas.

$\mathrm{O}$ único grupo que apresentou diferença significativa quanto ao número de meninos e meninas foi o grupo Abuso sexual, sendo constituído, na sua maior parte, pelo sexo feminino $(76,5 \%)$. Este dado é consoante com a literatura, que aponta que as meninas sofrem mais abuso sexual, enquanto que os meninos vivenciam mais episódios de agressões físicas (Asscher, van der Put, \& Stams, 2015). Estima-se que enquanto uma em cada cinco meninas são vítimas de abuso sexual, um em cada 20 meninos sofrem este tipo de violência (National Center for Victims of Crime, 2017).

Em relação às características do grupo abuso sexual, os resultados do presente estudo apontam que mais da metade das crianças experienciaram episódios recorrentes $(56,3 \%)$, a maioria deles perpetrados por familiares $(82,3 \%)$, do sexo masculino $(94,1 \%)$. Tais achados são corroborados pela literatura, a qual aponta que cerca de $80 \%$ dos casos de abuso sexual são perpetrados por pessoas próximas à vítima ( $\mathrm{Ad}$ vocacy Center, 2014) e do sexo masculino ( $\mathrm{Na}$ tional Center for Posttraumatic Stress Disorder, 2014). Na maioria dos casos do presente estudo, houve algum tipo de contato físico $(94,1 \%)$, com o uso de ameaça $(71,4 \%)$. Porém, a minoria dos casos obteve resultado positivo na perícia física $(8,8 \%)$. Tal informação é consoante com estudos que apontam que a maioria das alegações de abuso sexual contra crianças não deixa lesões físicas (Joki-Erkkila et al., 2014). Além disso, quando há positividade nos exames, estas evidências decorrem, na maior parte dos casos, de situações em que houve conjunção carnal ou quando a violência foi praticada com uso da força física, o que é mais frequente em casos de violência extrafamiliar.

Ainda, o grupo Abuso sexual foi o que apresentou os escores mais altos no item 34 do TSCC que avalia "não poder confiar nas pessoas porque elas podem querer manter relações sexuais", sendo que foram observadas diferenças significativas nesta variável em relação aos demais grupos. Até o momento não foram encontrados estudos que tenham avaliado a capacidade discriminante desta variável entre crianças vítimas de abuso sexual, embora tenha sido ressaltado que esse item merece ser foco de atenção clínica, podendo indicar a ocorrência de abuso sexual (Briere, 1996). Portanto, a partir desse achado, sugere-se a realização de mais estudos que investiguem a capacidade discriminante desta variável, tendo em vista que ela pode ser um potencial indicador da ocorrência de abuso sexual.

Quanto à capacidade discriminante de variáveis comumente associadas na literatura com a hipótese de abuso sexual, a maioria delas não apresentou significância estatística no presente estudo para diferenciar os grupos. Por exemplo, a variável de sintomas de estresse pós-traumático não apresentou diferença estatisticamente significativa entre os grupos, contrariando achados anteriores (Lanktree et al., 2008; Peixoto, 2012). Em estudo que investigou a presença de sintomas pós-traumáticos como ferramenta sensível para aferir a credibilidade de alegações de abuso sexual, o único indicador que apareceu como capaz de discriminar crianças com suspeita de abuso sexual daquelas sem essa suspeita foi a variável estresse pós-traumático, mensurada pelos instrumentos TSCC e TSCYC - Trauma Symptom Checklist for Young Children (Peixoto, 2012). Resultados semelhantes também haviam apontado a escala Estresse pós-traumático do TSCC como sensível para distinguir crianças vítimas de abuso sexual daquelas que não passaram por esta ocorrência (Lanktree et al., 2008). 
Uma das hipóteses para que a variável Sintomas de estresse pós-traumático não tenha apresentado diferença estatisticamente significativa no presente estudo é que as crianças vítimas de abuso sexual foram comparadas com um grupo de crianças que também passaram por outros eventos traumáticos, especificamente, por outros maus-tratos.

Com relação à variável Comportamentos sexuais, pesquisas realizadas apontaram para a importância de se avaliar a presença de comportamentos sexuais inadequados para o nível de desenvolvimento em crianças abusadas sexualmente, pois, supostamente, tais indicadores seriam manifestações típicas deste tipo de violência (Friedrich et al., 2001; Friedrich et al., 1997; Kendall-Tackett et al., 1993). Conforme estudo de normatização do CSBI, o instrumento é sensível para mensurar os efeitos do abuso sexual, embora os próprios autores tenham ressaltado que comportamentos sexuais também podem ser encontrados em outros casos em que a criança não foi sexualmente abusada, como por exemplo, quando foi exposta a eventos de vida estressantes ou apresenta diagnósticos psiquiátricos (Friedrich et al., 2001; Friedrich et al., 1992). Embora a presença de comportamentos sexuais seja comumente associada ao abuso sexual em crianças, no presente estudo essa variável não apresentou significância estatística para discriminar os grupos. Além disso, os comportamentos sexuais também foram identificados não apenas nas crianças do grupo Abuso sexual $(M=4,74)$ como nas crianças pertencentes tanto ao grupo Sintomas clínicos $(M=3,13)$ quanto ao grupo Maus-tratos $(M=5,86)$, inclusive com escores mais altos neste último grupo. Pesquisas anteriores também não haviam encontrado diferenças estatisticamente significativas nesta variável (Drach, Wientzen, \& Ricci, 2001; Peixoto, 2012). Por exemplo, em estudo conduzido a partir dos escores da escala total do CSBI, não foi possível discriminar crianças abusadas sexualmente daquelas que não passaram por esta experiência, evidenciando que a conclusão da ocorrência de abuso sexual a partir da presença de comportamentos sexuais inapropriados pode ser equivocada (Drach et al., 2001).
Ao mesmo tempo, quando conduzidas análises de diferenças entre os grupos nos nove domínios do CSBI foram observadas diferenças estatisticamente significativas em Intromissão sexual e em Conhecimento sexual, ambos com escores mais altos no grupo Abuso sexual, seguido pelo grupo Maus-tratos sem histórico de abuso sexual, e em Comportamento voyeurístico, com escores mais altos no grupo Maus-tratos sem histórico de abuso sexual, seguido pelo grupo Abuso sexual. Com relação a isso, autores já haviam salientado que o abuso sexual está mais relacionado a um conhecimento sexual inadequado do que a comportamentos sexuais problemáticos (Brilleslijper-Kater, Friedrich, \& Corwin, 2004). Todavia, pelo tamanho amostral do estudo, optou-se por incluir no modelo de regressão apenas o escore total do CSBI. Portanto, sugere-se que estudos futuros levem em consideração a análise individual de cada domínio do instrumento, tendo em vista que o abuso sexual pode estar mais diretamente relacionado a domínios específicos e não necessariamente a comportamentos sexuais problemáticos de uma maneira geral.

Comportamentos sexuais inadequados para o nível de desenvolvimento da criança apresentam um espectro muito amplo e podem assumir diferentes manifestações, como por exemplo, através de exibicionismo, comportamentos masturbatórios frequentes e em público, conhecimento e interesse sexual avançados para a idade, esforços para observar ou tocar os genitais de outras pessoas, comportamento sedutor, entre outros (Friedrich et al., 1997). Tais comportamentos podem estar presentes ou não em crianças vítimas de abuso sexual e diferenciarem-se tanto na intensidade como no tipo de sintoma observado (Hall, Mathews \& Pearce, 2002). Além disso, esta heterogeneidade pode ser justificada em função de peculiaridades intrínsecas à própria dinâmica do abuso sexual e/ou de circunstâncias adicionais (Friedrich et al., 2001). Há ainda que destacar que comportamentos sexuais inapropriados também são observados em crianças sem histórico de abuso sexual, como por exemplo, entre aquelas que vivenciaram abuso físico, emocional ou violência doméstica (Cha- 
ffin et al., 2008; Friedrich, 2007), o que também foi encontrado como resultado nesta pesquisa em que, inclusive, as crianças do grupo Maus-tratos obtiveram uma média mais alta no escore total do CSBI $(M=5,86)$ do que as crianças do grupo Abuso sexual $(M=4,74)$, conforme referido anteriormente. Embora no presente estudo não tenha sido encontrado diferenças entre os grupos com relação aos comportamentos sexuais entre as crianças de 8 a 12 anos, convém ressaltar que pesquisas sugerem que os escores mais altos nesta variável aparecem em crianças mais novas (Friedrich et al., 2001; Peixoto, 2012). Além disso, como o instrumento é respondido pelos próprios cuidadores, em alguns casos, estes podem não conseguir fornecer com exatidão a real situação da criança, ora sub ou superestimando sintomas (Friedrich et al., 2007).

No presente estudo, a variável Preocupações Sexuais foi a única que apresentou significância estatística para diferenciar as crianças sexualmente abusadas dos demais grupos. O modelo apresentou capacidade geral de classificar corretamente $69,6 \%$ dos casos. Escores elevados nesta escala já tinham sido referidos como sugestivos de abuso sexual (Briere, 1996). Entretanto, no estudo conduzido por Peixoto (2012), que, conforme mencionado anteriormente, um dos objetivos era avaliar a presença de sintomas pós-traumáticos como ferramenta sensível para aferir a credibilidade de alegações de abuso sexual, a variável Preocupações sexuais não apresentou significância estatística para diferenciar as crianças vítimas de abuso sexual do grupo sem esse histórico. Não foram encontradas outras pesquisas com estes objetivos para corroborar o achado encontrado no presente estudo. Apesar disso, este resultado chama a atenção para que esta variável possa ser mensurada em casos de suspeita de abuso sexual. Ademais, os escores desta escala são obtidos a partir das respostas fornecidas pela própria criança, ao contrário da variável Comportamentos sexuais mensurada pelo CSBI.

Por último, é importante destacar que foram excluídas das análises as crianças que pontuaram acima do ponto de corte do estudo de normatização do TSCC para sub e superestima- ção dos sintomas. Estas escalas de validade são um diferencial do instrumento, sobretudo no contexto forense, em que há uma preocupação maior com a exatidão e a validade das informações recebidas.

Uma das limitações na avaliação da sintomatologia desencadeada após o(s) episódio(s) de abuso sexual é a grande variabilidade existente entre o tempo transcorrido entre o último episódio de abuso sexual e a data da avaliação, pois, muitas vezes, as crianças acabam revelando os episódios tardiamente. Tal aspecto também foi observado na amostra, já que em $24,2 \%(n=9)$ das vítimas de abuso sexual, o último episódio possivelmente teria ocorrido há mais de um ano da data da coleta dos dados. A ausência de exatidão nas informações fornecidas pelos próprios participantes dificultou o controle desta variável, pois, em alguns casos, nem a criança nem seu responsável conseguiram precisar o período da ocorrência do(s) episódio(s) de abuso sexual e de outros maus-tratos.

Com relação às análises da amostra geral, convém ressaltar que houve diferença significativa no total da amostra quanto à distribuição de meninos e meninas, com maior prevalência do sexo feminino. Desse modo, uma amostra composta por mais participantes do sexo masculino auxiliaria a concluir com mais segurança quanto à presença ou não de diferenças significativas entre os sexos na manifestação clínica, uma vez que estudos relatam que a idade e o sexo da vítima impactam no quadro sintomatológico resultante dos abusos (Hébert, Lavoie, \& Blais, 2014; Ruiz, 2016).

\section{Considerações Finais}

Uma das maiores contribuições deste estudo foi investigar indicadores psicológicos e comportamentais como possíveis discriminantes não apenas entre crianças vítimas de abuso sexual e crianças sem qualquer histórico de vitimização, mas também entre crianças que passaram por outros tipos de maus-tratos. A variável Preocupações sexuais apresentou boa capacidade discriminante nesta amostra, sugerindo que altos escores nesta escala podem reforçar a hipótese 
de abuso sexual (Briere, 1996). A partir desse achado, recomenda-se que essa variável seja incluída, entre outros indicadores, nos procedimentos periciais de crianças com essa suspeita. Além disso, sugere-se que outras variáveis como os itens críticos do TSCC e os diferentes domínios do CSBI possam ser melhor averiguados em pesquisas futuras, uma vez que podem configurar como potenciais indicadores de abuso sexual ou revelar alguma situação específica que deva ser foco de atenção clínica ou necessite de investigações adicionais.

Apesar de algumas peculiaridades serem observadas na manifestação da sintomatologia de crianças vítimas de abuso sexual em comparação com vítimas de outros tipos de maus-tratos, pesquisadores reforçam que, mais importante do que o tipo de maltrato vivenciado, é a cronicidade, a severidade e as circunstâncias do evento (Jonkman, Verlinden, Bolle, Boer, \& Lindauer, 2013). Tais informações são importantes de serem levadas em consideração, especialmente no contexto forense, pois quando se trata de abuso sexual é preciso ficar claro que é um evento traumático cuja natureza varia ao longo de um contínuo de gravidade e as experiências das crianças podem ser completamente diferentes, influenciando, consequentemente, o impacto do evento para cada uma delas (Schaefer, Lobo, Brunnet, \& Kristensen, 2016).

De maneira geral, os resultados obtidos indicam a necessidade de cautela no estabelecimento de associações causais entre alterações comportamentais ou psicológicas e a hipótese de abuso sexual em crianças. Devido à grande complexidade e variabilidade entre os casos de abuso sexual, não há um quadro sintomatológico único e, portanto, basear a confirmação deste tipo de ocorrência em um único indicador ou em indicadores pouco fiáveis é um grande erro (Peixoto, 2012). Ao mesmo tempo, a inexistência de um quadro sintomatológico também não exclui a suspeita de abuso sexual, assim como a presença de sintomas pode ser decorrente de outros tipos de maus-tratos ou até mesmo uma reação ao próprio contexto de denúncia e do consequente processo judicial, independentemente da veracidade das alegações (Peixoto, 2012).
Mensurar o impacto do trauma e outras variáveis associadas pode ser útil para estimar os prejuízos desencadeados e corroborar outras evidências, aumentando a credibilidade da avaliação (Everson \& Faller, 2012). Porém, tais indicadores devem sempre ser interpretados dentro do contexto de avaliação e serem complementares à entrevista forense e nunca isoladamente para validar uma suspeita de abuso sexual.

$\mathrm{Na}$ prática pericial, o perito deve firmar suas conclusões em informações que possam ser sustentadas cientificamente (Ribeiro \& Peixoto, 2013). Nesse contexto, estabelecer o nexo causal entre a situação alegada e a sintomatologia manifestada é uma importante e difícil tarefa, pois sem o relato da criança ou outras evidências físicas e/ou biológicas nada é possível afirmar quanto à ocorrência ou não da situação abusiva (Peixoto, 2012; Peixoto et al, 2017; Pelisoli et al., 2016). Por conseguinte, é fundamental a consideração de hipóteses alternativas para o quadro sintomatológico avaliado e, dessa maneira, a investigação de outros possíveis eventos no histórico de vida e no desenvolvimento da criança é primordial (Schaefer et al., 2012).

Especialmente no contexto forense, é imprescindível a elaboração de uma bateria de instrumentos válidos que inclua dados provenientes de diferentes fontes de informação, a fim de que os achados possam ser comparados. Além disso, tendo em vista o valor probatório do relato da criança para o exame pericial e o consequente desfecho judicial, esforços devem continuar sendo empregados na capacitação dos profissionais e na adaptação de protocolos de entrevista forense válidos para este contexto e para esta população. Desta maneira, os profissionais poderão efetivamente aderir às recomendações de boas práticas na investigação do abuso sexual (Williams et al., 2014). A partir desse conjunto de indicadores, torna-se possível subsidiar com mais confiança e assertividade as conclusões periciais.

\section{Contribuições dos Autores}

Contribuição substancial no conceito e desenho do estudo: Luiziana Souto Schaefer; Christian Haag Kristensen. 
Contribuição na coleta de dados: Luiziana Souto Schaefer; Alice Einloft Brunnet; Beatriz de Oliveira Meneguelo Lobo; Janaína Núñez Carvalho.

Contribuição na análise e interpretação de dados: Luiziana Souto Schaefer; Alice Einloft Brunnet; Janaína Núñez Carvalho; Christian Haag Kristensen.

Contribuição para a preparação do manuscrito: Luiziana Souto Schaefer; Alice Einloft Brunnet; Janaína Núñez Carvalho; Christian Haag Kristensen.

Contribuição à revisão crítica, agregando conteúdo intelectual: Luiziana Souto Schaefer; Alice Einloft Brunnet; Beatriz de Oliveira Meneguelo Lobo; Janaína Núñez Carvalho; Christian Haag Kristensen.

\section{Conflitos de interesse}

Os autores declaram não ter conflito de interesse relacionado à publicação deste manuscrito.

\section{Referências}

Achenbach, T. M. (1991). Manual for the child behavior checklist/418 and 1991 profile. Burlington, VT: University of Vermont.

Achenbach, T. M. (2001). Manual for the child behavior checklist/618 and 2001 profile. Burlington, VT: University of Vermont.

Advocacy Center. (2014). Child sexual abuse. Retrieved from http://www.theadvocacycenter. org/adv abusewho.html

American Psychiatric Association. (2014). Diagnostic and statistical manual of mental disorders ( $5^{\text {th }}$ ed.). Washington, DC: Author.

Angelini, A. L., Alves, I. C. B., Custódio, E. M., \& Duarte, W. (1987). Manual das matrizes progressivas coloridas. São Paulo, SP: Casa do Psicólogo.

Angelini, A. L., Alves, I. C. B., Custódio, E. M., Duarte, W. F., \& Duarte, J. L. M. (1999). Matrizes progressivas coloridas de Raven: Escala especial. São Paulo, SP: Centro Editor de Testes e Pesquisa em Psicologia.

Asscher, J. J., van der Put, C. E., \& Stams, G. J. J. (2015). Gender Differences in the Impact of Abuse and neglect victimization on adolescent offending behavior. Journal of Family Violence, 30(2),215-225. doi: 10.1007/s 10896-014-9668-4
Associação Brasileira de Empresas de Pesquisa. (2015). Critério de Classificação Econômica Brasil. Recuperado em http://www.abep.org/ codigos-e-guias-da-abep

Bordin, I. A. S., Mari, J. J., \& Caeiro, M. F. (1995). Validação da versão brasileira do Child Behaviour Checklist (CBCL) (Inventário de Comportamento da Infância e da Adolescência): Dados preliminares. Revista da ABP, 17(2), 55-66. doi: 10.2471/BLT.07.043125

Briere, J. (1996). Trauma Symptom Checklist for Children (TSCC) professional manual. Odessa, FL: Psychological Assessment Resources.

Brilleslijper-Kater, S. N., Friedrich, W. N., \& Corwin, D. L. (2004). Sexual knowledge and emotional reaction as indicators of sexual abuse in young children: Theory and research challenges. Child Abuse \& Neglect, 28(10), 1007-1017. doi: 10.1016/j.chiabu.2004.06.005

Chaffin, M., Berliner, L., Block, R., Johnson, T. C., Friedrich, W. N., Louis, D. G., \& Madden, C. (2008). Report of the ATSA task force on children with sexual behavior problems. Child Maltreatment, 13(2), 199-218. doi: 10.1177/1077559507306718

Chaplin, T. M., \& Aldao, A. (2013). Gender differences in emotion expression in children: A meta-analytic review. Psychological Bulletin, 139(4), 735-765. doi: 10.1037/a0030737

Collin-Vézina, D., Daigneault, I., \& Hébert, M. (2013). Lessons learned from child sexual abuse research: Prevalence, outcomes, and preventive strategies. Child and Adolescent Psychiatry and Mental Health, 7-22. doi: 10.1186/1753-20007-22

Drach, K. M., Wientzen, J., \& Ricci, L. R. (2001). The diagnostic utility of sexual behavior problems in diagnosing sexual abuse in a forensic child abuse evaluation clinic. Child Abuse \& Neglect, 25(4), 489-503. doi: 10.1016/S01452134(01)00222-8

Everson, M. D., \& Faller, K. C. (2012). Base rates, multiple indicators, and comprehensive forensic evaluations: Why sexualized behavior still counts in assessments of child sexual abuse allegations. Journal of Child Sexual Abuse, 21, 45-71. doi: 10.1080/10538712.2012.642470

Faller, K. C. (2003). Understanding and assessing child sexual maltreatment $\left(2^{\text {th }}\right.$ ed.). Thousand Oaks, CA: Sage.

Friedrich, W. N. (2007). Children with sexual behavior problems. New York: W.W. Norton. 
Friedrich, W. N., Fisher, J. L., Dittner, C. A., Acton, R., Berliner, L., Butler, J., \& Wright, J. (2001). Child sexual behavior inventory: Normative, psychiatric, and sexual abuse comparisons. Child Maltreatment, 6(1), 37-49. doi: 10.1177/1077559501006001004

Friedrich, W. N., Grambsch, P., Damon, L., Hewitt, S. K., Koverola, C., Lang, R. A., ...Broughton, D. (1992). Child sexual behavior inventory: Normative and clinical comparisons. Psychological Assessment, 4(3), 303-311. doi: $10.1177 / 1077559501006001004$

Friedrich, W. N., Jaworski, T. M., Huxsahl, J., \& Bengtson, B. (1997). Dissociative and sexual behaviors in children and adolescents with sexual abuse and psychiatric histories. Journal of Interpersonal Violence, 12, 155-171. doi: $10.1177 / 088626097012002001$

Gava, L. L., Pelisoli, C., \& Dell'Aglio, D. D. (2013). A perícia psicológica em casos de suspeita de abuso sexual infanto-juvenil. Avaliação Psicológica, 12(2), 137-145. Recuperado em http://pepsic. bvsalud.org/pdf/avp/v12n2/v12n2a05.pdf

Guerra, C., Ocaranza, C., \& Weinberger, K. (2016). Searching for social support moderates the relationship between polyvictimization and externalizing symptoms: A brief report. Journal of Interpersonal Violence. doi: $10.1177 / 0886260516642293$

Habigzang, L. F., Borges, J. L., Dell'Aglio, D. D., \& Koller, S. H. (2010). Caracterização dos sintomas do Transtorno de Estresse Pós-Traumático (TEPT) em meninas vítimas de abuso sexual. Psicologia Clínica, 22(2), 27-44. doi: 10.1590/ S0103-56652010000200003

Hall, D. K., Mathews, F., \& Pearce, J. (2002). Sexual behavior problems in sexually abused children: A preliminary typology. Child Abuse \& Neglect, 26(3), 289-312. doi: 10.1016/S01452134(01)00326-X

Hamby, S., Finkelhor, D., \& Turner, H. (2014). Origine et développement du concept de polyvictimisation. Criminologie, 47(1), 11-15. doi: $10.7202 / 1024004$

Hébert, M., Lavoie, F., \& Blais, M. (2014). Post Traumatic Stress Disorder/PTSD in adolescent victims of sexual abuse: Resilience and social support as protection factors. Ciênc. Saúde Coletiva, 19(3), 685-694. doi: 10.1590/141381232014193.15972013

Irigaray, T. Q., Fonseca, R. P., Pacheco, J., Leite, J. C. C., Grassi-Oliveira, R., \& Kristensen, C.
H. (2013). Child maltreatment and later cognitive functioning: A systematic review. Psicologia: Reflexão \& Crítica, 26(2), 376-387. doi: 10.1590/S0102-79722013000200018

Joki-Erkkila, M., Niemi, J., \& Ellonen, N. (2014). Child sexual abuse - Medical statement conclusions in criminal legal process. Forensic Science International, 239, 31-26. doi: 10.1016/j. forsciint.2014.03.006

Jonkman, C. S., Verlinden, E., Bolle, E. A., Boer, F., \& Lindauer, J. L. (2013). Traumatic stress symptomatology after child maltreatment and single traumatic events: Different profiles. Journal of Traumatic Stress, 26(2), 225-32. doi: 10.1002/ jts.21792

Kendall-Tackett, K. A., Williams, L. M., \& Finkelhor, D. (1993). Impact of sexual abuse on children: A review and synthesis of recent empirical studies. Psychological Bulletin, 113(1), 164-180. doi: 10.1037/0033-2909.113.1.164

Lanktree, C. B., Gilbert, A. M., Briere, J., Taylor, N., Chen, K., Maida, C. A., \& Saltzman, W. R. (2008). Multi-informant assessment of maltreated children: Convergent and discriminant validity of the TSCC and TSCYC. Child Abuse \& Neglect, 32(6), 621-625. doi: 10.1016/j.chiabu. 2007.10 .003

Lobo, B., Brunnet, A., Ecker, K., Schaefer, L., Arteche, A., Gauer, G., \& Kristensen, C. (2015). Psychometric properties of the child posttraumatic cognitions inventory in a sample of brazilian children. Journal of Aggression, Maltreatment and Trauma, 24(8), 863-875. http://doi.org/10. 1080/10926771.2015.1043065

Lobo, B., Brunnet, A., Ecker, K., Schaefer, L., Arteche, A., \& Kristensen, C. (2015). CrossCultural Adaptation and Psychometric Properties of the Trauma Symptom Checklist for Children (TSCC) in a Sample of Brazilian Children: Preliminary Results. Journal of Child \& Adolescent Trauma. http://doi.org/10.1007/ s40653-0150044-1

Meiser-Stedman, R., Smith, P., Bryant, R., Salmon, K., Yule, W., Dalgleish, T., \& Nixon, R. D. V. (2009). Development and validation of the Child Post-Traumatic Cognitions Inventory (CPTCI). Journal of Child Psychology and Psychiatry, and Allied Disciplines, 50(4), 432-440. doi: 10.1111/j.1469-7610.2008.01995.x

Miele, A., \& Arteche, A. X. (2017). Tradução e Adaptação para o Português do Child Sexual Behavior Inventory. Manuscrito em preparação. 
National Center for Posttraumatic Stress Disorder. (2014). Child sexual abuse. Retrieved from http://www.ptsd.va.gov/public/types/violence/ child-sexual-abuse.asp

National Center for Victims of Crime (2017). Child sexual abuse statistics. Retrieved from https:// victimsofcrime.org/media/reporting-on-childsexual-abuse/child-sexual-abuse-statistics

Paolucci, E. O., Genuis, M. L., \& Violato, C. (2001). A meta-analysis of the published research on the effects of child sexual abuse. The Journal of Psychology, 135, 17-36. doi: $10.1080 / 00223980109603677$

Peixoto, C. E. (2012). Avaliação da credibilidade de alegações de abuso sexual de crianças: Uma perspetiva psicológica forense (Tese de doutorado, Universidade do Porto, Portugal).

Peixoto, C. E., Fernandes, R. V., Almeida, T. S., Silva, J. M., La Rooy, D., Ribeiro, C., ...Lamb, M. E. (2017). Interviews of Children in a Portuguese Special Judicial Procedure. Behavioral Sciences \& the Law, 35(3), 189-203. doi: 10.1002/bsl.2284

Pelisoli, C. L., Dell'Aglio, D. D., \& Herman, S. (2016). Sete erros na avaliação de abuso sexual contra crianças e adolescentes. In S. J. L. Vasconcelos \& V. de M. Lago (Eds.), $A$ psicologia jurídica e as suas interfaces: Um panorama atual (pp. 127-146). Santa Maria, RS: Editora da Universidade Federal de Santa Maria.

Portal Governo do Brasil, Direitos Humanos. (2017). Dia nacional contra abuso sexual de crianças e jovens é celebrado nesta quinta. Recuperado em http://www.brasil.gov.br/cidadania-ejustica/2017/05/dia-nacional-contra-abusosexual-de-criancas-e-jovens-e-celebrado-nestaquinta-18

Raven, J. C., Court, J. H., \& Raven, J. (1995). Manual for Raven's Progressive Matrices and Vocabulary Scales. Oxford: Oxford Psychologists Press.

Resolução n ${ }^{\circ} 466$ de 12 de dezembro de 2012. (2013, 13 jun.). Diário Oficial da União, p. 59.

Ribeiro, C., \& Peixoto, C. E. (2013). Avaliação psicológica forense de crianças alegadamente vítimas de abuso sexual. In T. Magalhães \& D. N. Vieira (Eds.), Abuso \& Negligência Série 2
- Agressões Sexuais: Intervenção Pericial Integrada (pp. 103-119). Maia, Portugal: Sociedade Portuguesa para o Estudo da Criança Abusada e Negligenciada.

Ruiz, E. (2016). Trauma symptoms in a diverse population of sexually abused children. Psychological Trauma: Theory, Research, Practice, and Policy, 8(6), 680-687. doi: 10.1037/tra0000160

Schaefer, L. S., Lobo, B. O. M., Brunnet, A. E. , Kristensen, C. H. (2016). Reações póstraumáticas em crianças: Como, por que e quais aspectos avaliar? Interação em Psicologia, 20(1), 112123. doi: 10.5380/psi.v20i1.30294

Schaefer, L. S., Rossetto, S., \& Kristensen, C. H. (2012). Perícia Psicológica no abuso sexual de crianças e adolescentes. Psicologia: Teoria e Pesquisa, 28, 227-234. doi: 10.1590/S010237722012000200011

Stoltenborgh, M., van IJzendoorn, M. H., Euser, E. M., \& Bakermans-Kranenburg, M. J. (2011). A global perspective on child sexual abuse: Meta-analysis of prevalence around the world. Child Maltreatment, 16(2), 79-101. doi: $10.1177 / 1077559511403920$

Van den Heuvel, L., \& Seedat, S. (2013). Screening and diagnostic considerations in childhood posttraumatic stress disorder. Neuropsychiatry, 3(5), 497-511. doi: 10.2217/npy.13.61

Wherry, J. N., Baldwin, S., Junco, K., \& Floyd, B. (2013). Suicidal thoughts/behaviors in sexually abused children. Journal of Child Sexual Abuse, 22(5), 534-551. doi: $10.1080 / 10538712.2013 .800938$

Williams, L. C. de A., Hackbarth, C., Aznar-Blefari, C., Padilha, M. da G. S., \& Peixoto, C. E. (2014). Investigação de suspeita de abuso sexual infantojuvenil: O Protocolo NICHD. Temas em Psicologia, 22(2), 415-432. doi: 10.9788/ TP2014.2-12

World Health Organization. (2002). World report on violence and health. Geneva: Author

Recebido: 12/07/2017

$1^{a}$ revisão: $16 / 08 / 2017$

Aceite final: 26/08/2017

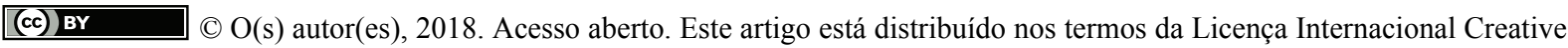
Commons Atribuição 4.0 (http://creativecommons.org/licenses/by/4.0/), que permite o uso, distribuição e reprodução sem restrições em qualquer meio, desde que você dê crédito apropriado ao(s) autor(es) original(ais) e à fonte, fornecer um link para a licença Creative Commons e indicar se as alterações foram feitas. 